\title{
Trabalho em turnos e dimensões sociais: Um estudo na indústria têxtil
}

\author{
Ana Isabel Ferreira \\ Isabel Soares Silva \\ Universidade do Minho
}

\begin{abstract}
Resumo
O trabalho em turnos tem sido associado a diversas consequências na vida dos trabalhadores. Este estudo analisa as consequências do trabalho em turnos ao nível familiar, social e organizacional e de que forma tais dimensões podem interferir na adaptação a estes horários. Foi utilizada uma amostra de 239 trabalhadores de uma empresa têxtil portuguesa, distribuídos pelos seguintes horários: convencional (08h-12h30/13h3017h), $1^{\circ}$ turno (06h/14h; turno da manhã), $2^{\circ}$ turno (14h/22h; turno da tarde) e $3^{\circ}$ turno (22h/06h; turno da noite). Na coleta de dados foi usado um questionário para avaliar, entre outros aspectos, a relação do horário de trabalho com a vida conjugal, a vida social ou o apoio da empresa. Os resultados apontam para uma preferência dos trabalhadores relativamente ao $1^{\underline{0}}$ turno, tendo este obtido os melhores scores em todos os aspectos. O horário convencional, por sua vez, apresenta os scores mais reduzidos. Genericamente, os resultados apontam para a importância de dimensões sociais na gestão do tempo de trabalho.
\end{abstract}

Palavras-chave: psicologia industrial; jornada de trabalho; trabalho em turnos; ambiente de trabalho; relações familiares

\begin{abstract}
Shiftwork and social dimensions: A study in the textile industry. Shiftwork has been associated to several consequences on the lives of workers. This study analyzes such consequences at the family, social and organizational levels, and how these dimensions may affect the workers' adaptation to these work schedules. A sample of 239 workers from a Portuguese textile factory was used in this study, subject to four types of work schedule: standard (8 a.m.-12:30 p.m./1:30 p.m.-5 p.m.), morning shift (6 a.m.-2 p.m.), evening shift (2 p.m.-10 p.m.) and night shift (10 p.m.-6 a.m.). Data were collected by means of a questionnaire which evaluated, among other things, the relationship between working time and conjugal life, social life or organizational support. Results indicate that workers have a preference for the morning shift which achieved the best scores in all aspects. The standard work schedule in turn shows the lowest scores. In a general way, results point to the importance of the social dimensions in the management of working time.
\end{abstract}

Keywords: industrial psychology; work hours; shift work; working conditions; family relations.

\section{Resumen}

Trabajo por turnos y dimensiones sociales: Un estudio en la industria textil. El trabajo por turnos se asocia con varias consecuencias para la vida de los trabajadores. Este estudio examina los efectos del trabajo por turnos a nivel familiar, social y organizacional, y de cómo estas dimensiones pueden interferir con la adaptación a estos horarios. Participaron 239 trabajadores de una empresa textil, distribuidos por horarios: convencional (08h-12h30/13h30-17h), turno de mañana (06h/14h), turno de tarde (14h/22h) y turno de noche (22/06h). Para la recogida de datos se utilizó un cuestionario para evaluar, entre otras cosas, la relación entre el tipo de horario y la vida conyugal y social, así como el apoyo de la empresa. Los resultados apuntan para una preferencia de los trabajadores del turno de mañana, teniendo estos trabajadores los mejores resultados en todos los aspectos. Por contrario, los trabajadores del horario convencional tienen los valores más bajos. En general, los resultados apuntan a la importancia de la dimensión social en la gestión del tiempo de trabajo.

Palabras clave: psicología industrial; horas de trabajo; trabajo por turnos; ambiente de trabajo; relaciones familiares

horário de trabalho fora dos padrões convencionais pode ser visto como um fenômeno social com repercussões ao nível da saúde e bem-estar dos trabalhadores (Presser,
1999). Independentemente da sua organização (turnos fixos, contínuos ou alternados), este tipo de horário pode acarretar problemas de saúde e perturbar o ambiente sociofamiliar de 
quem o pratica (Fischer \& Lieber, 2003).

Sendo o trabalho em turnos o objeto de várias investigações, estas tendem a focalizar-se em fatores de natureza individual em detrimento do estudo de variáveis de natureza situacional (Gadbois, 2004; Nachreiner, 1998). A este propósito, Handy (2010) também realça a limitação da investigação psicológica acerca do trabalho em turnos por esta centrar-se muito no próprio indivíduo, deixando para um plano secundário a esfera familiar (fator situacional). Mais recentemente, West, Mapedzahama, Ahern e Rudge (2012), referem também a tendência da investigação sobre o trabalho em turnos enfatizar os aspectos negativos, embora, tais horários, sob certas circunstâncias, possam trazer vantagens para os trabalhadores.

Com base nestas perspetivas, este estudo pretende contribuir para a compreensão do modo como os fatores de natureza familiar, social e organizacional podem influenciar a adaptação dos trabalhadores a um regime de trabalho em turnos. Nesse contexto, são revistos apenas os principais fatores suscetíveis de influenciar a adaptação de natureza situacional. Antes, porém, descreveram-se as principais consequências associadas ao trabalho por turnos a nível individual.

\section{Consequências do trabalho em turnos}

O trabalho em turnos não é peculiar apenas na sua forma de organização, mas também nas consequências que dele podem advir (Costa, 2004). O autor anteriormente citado refere que o trabalho em turnos apresenta consequências tanto ao nível intra como inter-individual, podendo estas serem moderadas por diversos aspectos, tais como, características individuais, estilo de vida, exigências do trabalho, políticas empresariais, relações familiares e contexto social. Sobre esta complexidade, veja-se, por exemplo, o estudo realizado por Saksvik-Lehouillier et al. (2012) com enfermeiras a trabalhar em horários alternados, onde se observou que diversas variáveis de natureza individual e situacional se encontravam relacionadas com a tolerância a este horário de trabalho.

O trabalho em turnos pode conduzir os indivíduos a determinados problemas/perturbações de saúde física e/ou psicológica como: problemas de sono, problemas ligados ao aparelho digestivo, problemas cardiovasculares, sintomatologia depressiva e/ou ansiosa, entre outras (Costa, 2004). Este tipo de perturbações surge também frequentemente acoplado a certas práticas como: maus hábitos alimentares, consumo de cigarro, café e fármacos (Silva, 2008). Monk e Folkard (1992) referem que o sistema circadiano humano é sincronizado, sobretudo, pelo ciclo "Claro-Escuro" e pelos contatos e rotinas sociais, enfatizando o efeito que o trabalho em turnos pode ter na perturbação dos ritmos circadianos do Homem, enquanto ser diurno.

O trabalho em turnos pode afetar social e familiarmente a vida dos trabalhadores podendo ser responsável pelo empobrecimento dos contatos sociais, assim como pela desorganização das rotinas familiares (Gadbois, 2004).

$\mathrm{Na}$ revisão de literatura elaborada por Demerouti, Geurts, Bakker e Euwema (2004), foi constatado que o trabalho em turnos abarca uma série de dificuldades com as quais os trabalhadores se podem confrontar, nomeadamente: diminuição da participação no voluntariado (Jamal, 1981), ausência na educação dos filhos (Diekmann et al., 1981) e diminuição da satisfação das esposas (Smith \& Folkard, 1993). Ainda na mesma revisão, é enfatizada a importância dos dias de fim de semana nos quais a manhã e a tarde parecem ser os períodos mais proveitosos pela maior participação em atividades sociais. Já durante a semana, é dada mais importância ao período noturno, embora o motivo se mantenha (Baer et al., 1981, 1985).

\section{Fatores situacionais da adaptação ao trabalho em turnos}

A investigação acerca do impacto do trabalho em turnos na esfera familiar tem sido posta à parte nos estudos realizados sobre a conciliação trabalho/família (Gadbois, 2004; Perrucci et al., 2007). Os autores anteriormente referidos defendem a realização de estudos que interliguem as escolhas familiares e individuais com as restrições estruturais do local de trabalho e o contexto social. Gadbois (2004) completa referindo que a realização de investigações neste contexto não se deve basear apenas nas percepções individuais (dos trabalhadores) e em dados quantitativos, mas devem complementar-se com estudos qualitativos que englobem os membros das famílias dos trabalhadores por turnos.

O trabalho em turnos pode ser entendido do ponto de vista do impacto familiar e social como uma "faca de dois gumes". $\mathrm{Se}$, por um lado pode possibilitar ao trabalhador ter mais tempo para se dedicar à família, ao cuidado dos filhos e às atividades sociais, por outro pode ter o efeito totalmente inverso (Shen \& Dicker, 2008).

No estudo realizado por Mott, Mann, McLoughlin e Warwick (1965), que envolveu a participação de mais de 1000 trabalhadores de turnos acerca dos efeitos do trabalho em turnos nas várias dimensões familiares, foi apurado que a vida doméstica seria a única dimensão que seria beneficiada com o regime de turnos. Nas restantes dimensões - companhia e proteção do cônjuge, momentos de convívio, tomadas de decisão conjuntas e relações sexuais - o trabalho em turnos parecia revelar-se menos vantajoso quando comparado com o trabalho diurno.

Ljoså e Lau (2009) realizaram um estudo em uma petrolífera que contou com a participação de 1398 homens e 299 mulheres. Os turnos eram contínuos, rotativos e tinham a duração de 12 horas, sendo que existiam equipes de trabalho em terra e em alto mar. Os resultados mostraram que, embora existissem trabalhadores que admitiam ter tempo suficiente para estar com o cônjuge, filhos, família e com os amigos, enfatizando o tempo prestado pela organização para atividades de lazer, existiam discrepâncias entre trabalhadores nestes níveis. Os indivíduos que trabalhavam "em terra" apresentavam scores mais reduzidos em relação ao tempo despendido com a família, cônjuge, filhos e amigos. Este resultado, segundo os autores, parece dever-se ao fato dos trabalhadores que laboravam "em terra" terem a necessidade de conciliar a sua vida laboral com a vida familiar/ conjugal e social.

Em entrevistas com membros de casais (27 trabalhadores e 17 esposas) em que o homem era trabalhador por turnos em uma indústria petroquímica, Handy (2010) concluiu que o isolamento 
vivido por parte das esposas pode, por vezes, ultrapassar os níveis dos próprios trabalhadores. $\mathrm{O}$ fato de estas mulheres passarem grande parte do seu tempo em casa e de não comparecerem a eventos sociais (por indisponibilidade do companheiro), pode exigir um ajustamento emocional ainda maior do que o dos seus maridos.

Demerouti et al. (2004) estudaram o impacto do trabalho em turnos no conflito trabalho-família relativamente às atitudes perante o trabalho e na saúde. Participaram neste estudo 2863 homens e 259 mulheres da Polícia Militar Holandesa, na maioria militares, sujeitos a um regime de trabalho em turnos (fixo e rotativo). Os dados revelaram que o trabalho em turnos pode surtir efeito no conflito trabalho-família, sendo que os trabalhadores de turnos fixos apresentaram scores mais elevados de conflito quando comparados com trabalhadores do regime rotativo. Estes resultados parecem estar relacionados, segundo os autores, com o fato de os trabalhadores dos turnos fixos estarem permanentemente impossibilitados de participarem em determinadas atividades, tanto familiares (horas das refeições, aniversários, etc.), como sociais (atividades recreativas, estar com os amigos, etc.).

A percepção de suporte organizacional sofre variações através da presença (ou não) de feedback, seja este através de recompensas ou simples elogios e dependendo da sua frequência e intensidade (Paschoal, Torres, \& Porto 2010). O suporte do contexto organizacional parece ser, assim como o contexto social e familiar, um importante fator situacional na adaptação ao trabalho em turnos (Nachreiner, 1998).

Ao nível do contexto organizacional, além do desenho do próprio sistema em turnos (ex., Knauth, 1996), a investigação aponta ainda para a relevância de uma participação dos trabalhadores, do ponto de vista da adaptação ao trabalho em turnos, a qual pode passar, entre outros aspectos, pela participação dos trabalhadores no desenho dos sistemas de turnos ou na escolha dos turnos em que gostariam de trabalhar (Silva, 2008). No estudo de Camerino et al. (2010), com 664 profissionais de enfermagem (98\% dos quais mulheres), além de ter sido observado um impacto diferenciado dos diferentes horários de trabalho no conflito trabalho-família, observou-se também que tal impacto era influenciado pela presença ou não de uma cultura organizacional preventiva desse tipo de conflito.

\section{Objetivos do estudo}

A presente investigação pretende analisar as consequências do trabalho em turnos ao nível familiar, social e organizacional e de que forma tais dimensões podem interferir na adaptação a estes horários. $\mathrm{O}$ estudo apresentado diz respeito à última fase de um projeto de investigação maior cujo propósito central era o de contribuir para o aprofundamento da compreensão dos aspectos familiares e sociais do trabalho em turnos e compreendia uma primeira fase exploratória (realização de entrevistas a casais da empresa onde decorreu o estudo em que pelo menos um dos membros do casal era trabalhador por turnos) e uma segunda fase de aplicação de um questionário, a qual é descrita no presente trabalho.

\section{Método}

\section{Instrumentos}

$\mathrm{Na}$ coleta dos dados foi utilizado um questionário estruturado, o qual abordava várias dimensões que passamos a descrever. Assim, e para efeitos de caracterização da amostra, foram requeridos os seguintes dados: idade, sexo, nível de escolaridade e estado civil. Ao nível da situação familiar foi questionada a existência de filhos, e em caso afirmativo, o seu número e respectivas idades. Foram ainda solicitadas informações acerca da situação profissional dos participantes, nomeadamente tempo de serviço na empresa e tempo de serviço no horário de trabalho atual.

Para avaliar a relação entre horário de trabalho e a esfera social, dimensão que doravante se passou a designar de "Vida fora da Empresa", foi utilizada uma escala adaptada de Silva (2008), desenvolvida para um contexto semelhante com base em Silva, Azevedo e Dias (1994) e Bohle e Tilley (1998). Esta escala é composta por seis itens, sendo dois deles os seguintes: "o seu horário de trabalho deixa-lhe tempo suficiente para estar com a família e os amigos mais chegados" e "o seu horário de trabalho deixa-lhe tempo para descansar convenientemente". O valor de alfa de Cronbach encontrado no estudo de Silva (2008) com mais de 700 trabalhadores têxteis foi de 0,91 .

Para avaliar a satisfação geral dos trabalhadores em relação ao horário de trabalho, foi utilizada uma escala desenvolvida por Silva (2008) para um contexto de trabalho semelhante, constituída por sete itens, com uma alfa de Cronbach de 0,89 que compreendia itens como "sente-se completamente satisfeito com o horário de trabalho que tem" e "aconselha o seu horário de trabalho a um familiar ou amigo próximo".

A escala de "Suporte da Empresa" foi também desenvolvida por Silva (2008) com base em entrevistas exploratórias a trabalhadores de empresas têxteis realizadas pela autora e com base em Eisenberger, Huntington, Hutchison e Sowa (1986). A escala pretende avaliar a percepção dos trabalhadores em relação ao modo como consideram que a empresa gere as questões relacionadas com os horários de trabalho. Esta era, na sua versão original, constituída por quatro itens com um alfa de Cronbach superior a 0,80 , sendo que, no âmbito do presente estudo, se procedeu à introdução de um novo item - "quando é necessário mudar pessoal entre os turnos, a empresa preocupase em informar os trabalhadores". A opção de introduzir este item partiu da análise das entrevistas (primeira fase do projeto), onde pareceu pertinente a existência de uma questão direcionada para a comunicação dentro da organização. São abarcados itens como: "a empresa faz o possível para colocar os trabalhadores nos turnos que eles preferem" e "a empresa tem em conta a preferência das pessoas por um ou outro turno na altura em que elas são admitidas".

De forma a avaliar a percepção do "Suporte Familiar/ Amigos" relativamente a questões relacionadas com o horário de trabalho, foi utilizada uma escala adaptada de Silva (2008) a partir de Caplan, Cobb, French, Van Harrison e Pinneau (1975). Esta escala é constituída por quatro itens dos quais fazem parte "Quando tem dificuldades criadas pelo seu horário de trabalho, 
pode contar sempre com a sua família e os seus amigos para o(a) ajudar a resolvê-las" e "é fácil para você falar com a sua família e os seus amigos quando precisa de ajuda em problemas provocados pelo seu horário de trabalho". O valor de alfa de Cronbach encontrado foi de 0,93.

As escalas que se designaram como "Vida Familiar" e "Vida Conjugal" foram desenvolvidas no âmbito do presente estudo e pretendiam avaliar, respectivamente, a conciliação entre o horário de trabalho e a vida familiar e conjugal. A escala relativa à "Vida Conjugal" diz respeito à influência que o trabalho em turnos pode ter na vida de casal e integra quatro itens. Um exemplo de um dos itens é "o seu horário de trabalho deixa-lhe tempo suficiente para estar com o/a seu/sua companheiro/a". Deveriam responder às questões desta escala apenas as pessoas que fossem casadas ou vivessem em união estável no momento da coleta de dados. A escala relativa à "Vida Familiar" se relacionava com a influência que os horários de trabalho têm na vida familiar - "o seu horário de trabalho deixa-lhe mais tempo para se dedicar ao papel de mãe/pai” constitui um dos quatro itens que integra a referida escala. Apenas deveriam responder a estas questões, os indivíduos que tivessem, atualmente, filhos menores de 18 anos morando consigo. Os itens destas escalas foram elaborados com base nos resultados das entrevistas, assim como em material bibliográfico escolhido para o efeito (Kopelman, Greenhaus, \& Connolly, 1983; Mott et al., 1965).

Em termos da escala de resposta, foi utilizada uma escala do tipo Likert com cinco pontos (1 - Discordo Totalmente até 5 - Concordo Totalmente). Para todos os casos, quanto maiores os scores obtidos, maior a percepção da variável em avaliação.

\section{Procedimentos}

Os dados foram recolhidos numa empresa têxtil, do ramo das algodoeiras, situada no norte de Portugal. A empresa contava, no momento da recolha dos dados, com um total de 398 trabalhadores cuja quantidade que trabalhava nos setores produtivos era de 361 , distribuídos pelos vários turnos da seguinte forma: $1^{\mathrm{o}}$ Turno (06h-14h) $-81 ; 2^{\mathrm{O}}$ Turno (14h-22h) - 79; $3^{\mathrm{o}}$ Turno $(22 \mathrm{~h}-06 \mathrm{~h})$ - 50. Os restantes 151 trabalhavam no horário considerado convencional (08h-12h30/13h30-17h).

Foram entregues 292 questionários, distribuídos por um investigador entre os trabalhadores dos turnos. Juntamente com o questionário estava presente uma carta de apresentação onde eram dadas informações acerca do estudo, assim como do preenchimento do questionário. Para que fosse garantido o anonimato assim como a confidencialidade total dos dados recolhidos, foi também dado a cada trabalhador um envelope devidamente etiquetado (endereçado à equipe de investigação) no qual estes deveriam depositar o questionário após o seu preenchimento. $\mathrm{O}$ envelope deveria, então, ser colocado numa caixa que foi situada no local da recolha de dados para o devido efeito.

Dos 292 questionários entregues foram devolvidos 268, o que corresponde a uma taxa de resposta de $91,8 \%$. No entanto, após a análise dos valores omissos e tendo em conta as orientações de Bryman e Cramer (2003), foram eliminados 27 questionários por apresentarem valores omissos superiores a $10 \%$. Foram ainda eliminados outros dois por não terem revelado qual o seu horário de trabalho. Deste modo, a amostra final ficou constituída por 239 trabalhadores.

\section{Amostra}

Como é possível analisar na Tabela 1, dos 239 trabalhadores, $53 \%$ eram do sexo feminino. Não obstante uma distribuição relativamente equitativa em função do sexo considerando o total da amostra, verificaram-se assimetrias quando se considerou o tipo de horário. Com efeito, as mulheres predominavam no horário convencional (aproximadamente 80\%), eram cerca de um terço no $1^{\underline{o}}$ e no $2^{\underline{o}}$ turno e estavam ausentes no $3^{\underline{o}}$. O nível de escolaridade era relativamente baixo, sendo que apenas cerca de 5\% tinha um nível de escolaridade superior ao ensino básico. A maioria era casada ou vivia em união estável (77\%) e tinha filhos (78\%) dos quais 71,9\% possuíam filhos com idade menor que 18 anos. A idade média se situava nos 39,7 anos $(D P=$ $8,7)$. A antiguidade média na empresa era, em todos os horários de trabalho, superior a 12 anos, enquanto que a antiguidade média no horário atual era, no mínimo, aproximadamente de 8 anos ( $2^{\underline{0}}$ e $3^{\underline{0}}$ turnos). Todos os trabalhadores se encontravam trabalhando na área da produção da empresa (por exemplo, tecelagem, confeção).

\section{Análise das medidas}

De modo a analisar as propriedades psicométricas das medidas utilizadas, foi realizada, em um momento inicial a análise fatorial exploratória com rotação varimax para aquelas medidas que foram desenvolvidas ou alteradas no âmbito do estudo, ou seja, as relativas à conciliação entre horário de trabalho e a "Vida Conjugal", a "Vida Familiar" e o "Suporte da Empresa”. Nos três casos foram considerados, primeiramente, os resultados obtidos nos testes KMO e esfericidade de Bartlett, os quais permitiram prosseguir com a análise fatorial exploratória (Pestana \& Gageiro, 2005).

No caso da medida relativa ao "Suporte da Empresa", foi extraído um fator com valor próprio superior a 1, que explicava $68,8 \%$ da variância total, tendo todos os itens obtido pesos de saturação maiores que 0,50 , nomeadamente compreendidos entre 0,81 e 0,85. De acordo com Pestana e Gageiro (2005), são considerados significativos os pesos de saturação com valores superiores a 0,50. Em termos de fidedignidade (análise da consistência interna), o valor do alfa de Cronbach obtido foi de 0,89, o que, segundo os autores citados, é considerado "bom".

A análise fatorial exploratória para a escala relativa à "Vida Conjugal" indicou igualmente a obtenção de um fator com valor superior a 1, o qual explicava $63,57 \%$ da variância total. No entanto, um dos itens "discute mais com o/a seu/sua companheiro(a) devido ao seu horário de trabalho" obteve um peso fatorial igual a 0,11 , o que, segundo Pestana e Gageiro (2005), constitui uma saturação considerada "inaceitável”. Os restantes itens obtiveram pesos fatoriais entre 0,90 (item 3 ) e 0,93 (item 2), o que respeita as recomendações dos autores já citados.

Em relação à fidedignidade (análise da consistência interna), o valor do alfa de Cronbach sem o contributo do item 4 foi de 0,92, o que pode ser considerada como "muito boa" (Pestana \& Gageiro, 2005); no entanto, com a presença deste item, o valor descia para 0,72 (considerada "razoável"). Considerando 
Tabela 1

Caracterização da Amostra

\begin{tabular}{|c|c|c|c|c|c|}
\hline Variável & $\begin{array}{c}\text { Horário } \\
\text { Convencional }\end{array}$ & $1^{\circ}$ Turno & $2^{\underline{0}}$ Turno & $3^{-0}$ Turno & TOTAL* \\
\hline \multirow[b]{2}{*}{ Colaboradores } & \multicolumn{5}{|c|}{$N(\%)$} \\
\hline & $117(49,0 \%)$ & $46(19,2 \%)$ & $46(19,2 \%)$ & $30(12,6 \%)$ & $239(100 \%)$ \\
\hline Sexo & \multicolumn{5}{|c|}{$N(\%)$} \\
\hline Masculino & $25(21,4 \%)$ & $29(63,0 \%)$ & $31(67,4 \%)$ & $28(100 \%)$ & $113(47,3 \%)$ \\
\hline Feminino & $92(78,6 \%)$ & $17(37,0 \%)$ & $15(32,6 \%)$ & - & $124(52,7 \%)$ \\
\hline Total & & & & & $237(100 \%)$ \\
\hline \multicolumn{6}{|l|}{ Idade (anos) } \\
\hline$M(D P)^{* *}$ & $40,5(7,8)$ & $38,8(9,1)$ & $38,3(10,0)$ & $40,1(9,1)$ & $39,7(8,7)$ \\
\hline Estado Civil & \multicolumn{5}{|c|}{$N(\%)$} \\
\hline Solteiro(a) & $14(12,5 \%)$ & $9(19,6 \%)$ & $13(28,9 \%)$ & $7(25,0 \%)$ & $43(18,6 \%)$ \\
\hline Casado(a)/União Estável & $90(80,4 \%)$ & $35(76,1 \%)$ & $31(68,9 \%)$ & $21(75,0 \%)$ & $177(76,6 \%)$ \\
\hline Viúvo(a) & $5(4,5 \%)$ & - & - & - & $5(2,2 \%)$ \\
\hline Separado(a)/Divorciado(a) & $3(2,7 \%)$ & $2(4,3 \%)$ & $1(2,2 \%)$ & - & $6(2,6 \%)$ \\
\hline Total & & & & & $231(100 \%)$ \\
\hline Filho(s) & \multicolumn{5}{|c|}{$N(\%)$} \\
\hline Filhos menores de 18 anos & $66(71,0 \%)$ & $24(75,0 \%)$ & $23(69,7 \%)$ & $15(75,0 \%)$ & $128(71,9 \%)$ \\
\hline Filhos maiores de 18 anos & $27(29,0 \%)$ & $8(25,0 \%)$ & $10(30,3 \%)$ & $5(25,0 \%)$ & $50(28,1 \%)$ \\
\hline Total & & & & & $178(100 \%)$ \\
\hline Familiar(es) num raio de $5 \mathrm{~km}$ & \multicolumn{5}{|c|}{$N(\%)$} \\
\hline Sim & $108(93,1 \%)$ & $40(87,0 \%)$ & $42(95,5 \%)$ & $27(90,0 \%)$ & $217(91,9 \%)$ \\
\hline Não & $8(6,9 \%)$ & $6(13,0 \%)$ & $2(4,5 \%)$ & $3(10,0 \%)$ & $19(8,1 \%)$ \\
\hline Total & & & & & $236(100 \%)$ \\
\hline
\end{tabular}

*Dada a existência de valores omissos em certas variáveis, o $N$ obtido pode ser diferente do tamanho da amostra $(N=239)$

**M (Média), DP (Desvio Padrão)

o conjunto dos dados, decidiu-se eliminar este item da escala.

Por fim, e no que diz respeito à escala "Vida Familiar", os dados obtidos através da análise fatorial apontaram para a extração de um único fator com valor superior a 1, explicando $60,63 \%$ da variância total. Através do cálculo dos pesos fatoriais foi possível apurar que o item 3 "o seu horário de trabalho não lhe permite passar com a sua familia tanto tempo quanto gostaria" possui um peso fatorial igual a 0,39 , não cumprindo assim os critérios propostos por Pestana e Gageiro (2005). À semelhança do caso anterior, também foi calculado para esta escala o valor do alfa de Cronbach sem a presença do referido item. A partir dos dados obtidos, verificou-se que a sua exclusão seria uma opção a considerar, uma vez que aumentava o valor do alfa de Cronbach de 0,75 para 0,85 .

Nas restantes escalas - "Vida fora da Empresa", "Satisfação com o Horário de Trabalho" e "Suporte Familiar/Amigos" - não foram realizadas análises fatoriais, uma vez que estas já haviam sido submetidas a este procedimento, bem como a análises fatoriais confirmatória, no trabalho de Silva (2008) com uma amostra idêntica. Não obstante, foram calculados os valores de consistência interna (alfa de Cronbach) encontrados neste estudo, os quais se encontram apresentados na Tabela 2. Essa Tabela também reúne os valores encontrados nas restantes escalas.

Todas as análises estatísticas realizadas para este estudo foram feitas com o auxílio do programa informático Statistical
Package for Social Sciences, SPSS, versão 17.0, em programa Windows.

\section{Resultados}

Em uma primeira fase, foram efetuados testes de correlação a fim de apurar a relação entre as variáveis em estudo, encontrando-se os resultados expressos na Tabela 2:

Por meio da análise da tabela 2 , é possível constatar que, excetuando a relação entre a "Vida Familiar" e o "Suporte Familiar/Amigos", existem relações estatisticamente significativas entre as restantes variáveis.

Uma vez que era pretendido avaliar se existiam diferenças estatisticamente significativas entre quatro grupos (horários de trabalho), em função das variáveis já referidas, o teste paramétrico utilizado foi a ANOVA unifatorial. Quando os pressupostos para a sua realização não eram verificados (Field, 2005), realizouse o teste não paramétrico Kruskal-Wallis. Esta última opção apenas não foi aplicada às variáveis relativas à "Satisfação com o Horário de Trabalho" e ao "Suporte da Empresa". No entanto, ambos os testes indicam apenas se existem diferenças estatisticamente significativas entre os grupos, não discriminando entre quais. Para esse efeito, e quando necessário, foi realizado o teste Post-Hoc de Scheffé. Como refere Maroco (2010), esse é um teste que realiza comparações múltiplas de médias, indicando, 
Tabela 2

Matriz de Correlações entre as Variáveis em Estudo e Valores Alfa de Cronbach das Medidas Utilizadas (Informação entre Parêntesis)

\begin{tabular}{|c|c|c|c|c|c|c|}
\hline & 1 & 2 & 3 & 4 & 5 & 6 \\
\hline \multicolumn{7}{|l|}{ 1. Vida fora Empresa $(0,89)$} \\
\hline 2. Satisfação Horário Trabalho $(0,85)$ & $0,56^{* * *}$ & & & & & \\
\hline 3. Suporte Empresa $(0,89)$ & $0,40 * * *$ & $0,36 * * *$ & & & & \\
\hline 4. Suporte Família/Amigos $(0,92)$ & $0,30 * * *$ & $0,34 * * *$ & $0,20 * *$ & & & \\
\hline 5. Vida Conjugal $(0,92)$ & $0,49 * * *$ & $0,60 * * *$ & $0,23 * *$ & $0,20 * *$ & & \\
\hline 6. Vida Familiar $(0,85)$ & $0,59 * * *$ & $0,60 * * *$ & $0,40 * * *$ & 0,09 & $0,62 * * *$ & \\
\hline
\end{tabular}

$* * p<0,01 ; * * * p<0,001$

assim, os grupos entre os quais existem diferenças. Para as variáveis para as quais foi utilizado o teste de Kruskal-Wallis e que obtiveram resultados estatisticamente significativos, foram realizados, de acordo com as recomendações de Field (2005), testes Mann-Whitney com correção de Bonferroni (o valor da significância é dividido pelo número de testes efetuados, neste caso, $0,05 / 6=0,008)$.

Realizaram-se ainda testes de Qui-Quadrado no sentido de analisar a existência de diferenças entre os vários horários de trabalho e variáveis demográficas e familiares, designadamente quanto ao "sexo", "estado civil", "presença de filhos" e "presença de familiares num raio de $5 \mathrm{~km}$ de distância". Apenas no caso do sexo foram encontradas diferenças estatisticamente significativas na composição dos grupos em função dos horários de trabalho ( $\chi 2(3)=74,7, p<0,001)$ sendo que, o horário convencional, ao contrário dos restantes, é composto maioritariamente por mulheres.

"Satisfação com o horário de trabalho" e "Suporte da Empresa"

Como se pode constatar na Tabela 3, existem diferenças estatisticamente significativas na "Satisfação com o Horário de Trabalho" e a percepção de "Suporte da Empresa".

Tabela 3

Comparação dos Turnos em Função da "Satisfação com o Horário de Trabalho" e "Suporte da Empresa"

\begin{tabular}{|c|c|c|c|c|c|}
\hline & $\begin{array}{l}\text { Horário Convencional } \\
\qquad(n=117) \\
\text { Média }(D P)\end{array}$ & $\begin{array}{c}1^{\circ} \text { Turno } \\
(n=46) \\
\text { Média }(D P)\end{array}$ & $\begin{array}{c}2^{\underline{\mathrm{o}}} \text { Turno } \\
(n=46) \\
\text { Média }(D P)\end{array}$ & $\begin{array}{c}3^{\underline{0}} \text { Turno } \\
(n=30) \\
\text { Média }(D P)\end{array}$ & $F(3,235)$ \\
\hline Satisfação Horário Trabalho & $3,62(0,85)$ & $4,24(0,53)$ & $3,95(0,80)$ & $3,84(0,66)$ & $7,59 * * *$ \\
\hline Suporte da Empresa & $2,82(0,90)$ & $3,52(0,68)$ & $3,06(0,95)$ & $3,38(0,94)$ & $8,49 * * *$ \\
\hline
\end{tabular}

$* * * p<0,001$

A partir do teste Post-Hoc de Scheffé foi possível constatar que existem diferenças estatisticamente significativas entre o horário convencional e o $1^{\underline{0}}$ turno em relação à "Satisfação com o Horário de Trabalho", sendo que os trabalhadores do $1^{\circ}$ turno apresentaram maiores níveis de satisfação com o horário de trabalho, quando comparados com trabalhadores do horário convencional.

A partir das comparações múltiplas foi também possível constatar que existem diferenças estatisticamente significativas entre o horário convencional e o $1^{\underline{0}}$ e $3^{\underline{0}}$ turnos, em relação ao “Suporte da Empresa”. Especificamente, os trabalhadores do $1^{\mathrm{O}}$ turno apresentam maior concordância em relação à forma como a empresa lida com os problemas relacionados com os horários de trabalho, quando comparados com o horário convencional. O $3^{\underline{0}}$ turno também apresentou melhor apreciação no "Suporte da Empresa" em relação ao horário convencional.

"Vida Fora da Empresa", "Suporte Familiar/Amigos", "Vida Conjugal" e "Vida Familiar"
Como é possível visualizar através da Tabela 4, apenas na dimensão "Suporte Familiar/Amigos" não foram encontradas diferenças estatisticamente significativas entre os diferentes horários.

A realização de testes Mann-Whitney com correção Bonferroni relativamente à dimensão "Vida fora da Empresa" indicou a existência de diferenças estatisticamente significativas entre o horário convencional e o $1^{\underline{0}}, 2^{\underline{0}}$ e $3^{\underline{0}}$ turnos. Foram também encontradas diferenças estatisticamente significativas entre trabalhadores do $1^{\underline{0}}$ e do $2^{\underline{o}}$ turnos.

Os dados revelam que os trabalhadores do $1^{\underline{0}}, 2^{\underline{0}}$ e $3^{\underline{0}}$ turnos avaliaram como melhor a sua "Vida fora da Empresa" (participação em atividades sociais e de lazer) em relação aos indivíduos do horário convencional. A "Vida fora da Empresa" foi ainda melhor avaliada pelos trabalhadores do $1^{\underline{0}}$ turno em relação aos do $2^{\underline{0}}$ turno.

A realização dos testes Mann-Whitney com correção Bonferroni relativamente à dimensão "Vida Conjugal", 
Tabela 4

Comparação dos Turnos em Função da "Vida Fora da Empresa”, "Suporte Familiar/Amigos", "Vida Conjugal” e "Vida Familiar"

\begin{tabular}{|c|c|c|c|c|c|}
\hline & $\begin{array}{c}\text { Horário } \\
\text { Convencional } \\
(n=117) \\
\text { Ordem Média }\end{array}$ & $\begin{array}{c}\mathbf{1}^{\mathbf{0}} \text { Turno } \\
\quad(n=46) \\
\text { Ordem Média }\end{array}$ & $\begin{array}{c}2^{\mathbf{0}} \text { Turno } \\
\quad(n=46) \\
\text { Ordem Média }\end{array}$ & $\begin{array}{c}\mathbf{3}^{\mathbf{0}} \text { Turno } \\
\quad(n=30) \\
\text { Ordem Média }\end{array}$ & $\chi^{2}(3)$ \\
\hline Vida fora Empresa & 84,32 & 174,18 & 138,28 & 148,03 & $68,01 * * *$ \\
\hline Suporte Familiar/Amigos & 112,33 & 131,73 & 119,98 & 131,95 & 3,84 \\
\hline Vida Conjugal & 79,31 & 116,59 & 85,48 & 66,33 & $19,10 * * *$ \\
\hline Vida Familiar & 55,90 & 94,98 & 55,36 & 66,81 & $21,58 * * *$ \\
\hline
\end{tabular}

$* * * p<0,001$

encontrou diferenças estatisticamente significativas entre o horário convencional e o $1^{\underline{0}}$ turno. Foram ainda encontradas diferenças estatisticamente significativas entre os trabalhadores do $1^{\underline{0}}$ e $3^{\underline{0}}$ turnos. Concretamente, os trabalhadores do $1^{\underline{0}}$ turno apresentaram uma melhor conciliação entre o horário de trabalho e a "Vida Conjugal" em relação aos trabalhadores do horário convencional. A interface horário de trabalho/vida conjugal foi ainda melhor avaliada pelos trabalhadores do $1^{\underline{0}}$ turno em relação aos do $3^{0}$ turno.

Através da realização dos testes Mann-Whitney com correção Bonferroni no que toca à dimensão "Vida Familiar" foram encontradas diferenças estatisticamente significativas entre o horário convencional e o $1^{\underline{0}}$ turno. Existiram também diferenças estatisticamente significativas entre trabalhadores do $1^{\underline{0}}$ e $2^{\underline{0}}$ turnos em função da "Vida Familiar". Especificamente, os trabalhadores do $1^{\circ}$ turno avaliaram como melhor a conciliação feita entre o horário de trabalho e a vida familiar quando comparados com trabalhadores tanto do horário convencional como do $2^{\underline{0}}$ turno.

\section{Discussão dos resultados}

Com esta investigação, pretendeu-se analisar as consequências do trabalho em turnos ao nível familiar, social e organizacional e de que forma tais dimensões podem interferir na adaptação a estes horários. Os resultados obtidos indicam a existência de diferenças entre os vários horários ao nível da "Satisfação com o Horário de Trabalho", "Suporte da empresa" aos trabalhadores, "Vida fora da Empresa" (participação em atividades de natureza social e lazer), "Vida Conjugal" e "Vida Familiar".

O maior número de diferenças encontradas foi ao nível do horário convencional e do $1^{\underline{0}}$ turno (turno da manhã fixo), sendo que o último apresentou resultados superiores em todas as dimensões avaliadas.

Apesar de o horário convencional constituir o horário de trabalho tido como normal, os dados não apontam para que este seja, por esse motivo, mais vantajoso ao nível das esferas social e familiar/conjugal, assim como ao nível da satisfação com o horário de trabalho. No entanto, é importante sublinhar que o horário convencional se diferencia dos restantes horários de trabalho em termos da sua composição ao nível do sexo, sendo constituído maioritariamente por mulheres. Esta diferença na composição dos grupos deverá ser tida em conta na interpretação das comparações entre este horário e os restantes nas variáveis avaliadas, dadas as assimetrias que continuam existindo entre homens e mulheres em termos do trabalho doméstico/ responsabilidades familiares. Por exemplo, segundo informação disponível na página da Comissão para a Igualdade no Trabalho e no Emprego Portuguesa (2011), as mulheres, em 2011, consumiam mais 16 horas por semana quando comparadas com os homens, a realizar tarefas relacionadas com a vida doméstica tais como prestação de cuidados a crianças e familiares. Tais assimetrias podem contribuir para dificuldades acrescidas na gestão do tempo de trabalho e outras esferas da vida por parte das mulheres. Além do aspecto anterior, o fato de o horário convencional se caracterizar por ter um período de laboração de manhã e de tarde, de segunda a sexta-feira, acaba por deixar, em comparação com outros turnos (em especial o da manhã e o da noite), pouco tempo livre para dedicar a atividades de natureza familiar e/ou social. A investigação realizada por West et al. (2012) com enfermeiras observou que o horário de trabalho por turnos constituía para algumas profissionais uma vantagem na gestão de aspectos sociais e familiares pelo tempo livre e flexibilidade que proporcionava nos dias de folga (por exemplo, poder ir ao banco). Tais aspectos poderão também ajudar a compreender os melhores resultados observados no turno da manhã.

Com efeito, a avaliação que os trabalhadores do $1^{\underline{0}}$ turno fazem do suporte prestado pela empresa, assim como o nível de satisfação com o horário de trabalho que apresentam, poderão estar relacionados com o fato de estes indivíduos terem a possibilidade de melhor satisfazer as necessidades relacionadas com as esferas social e familiar/conjugal. Este turno tem início às 6h da manhã, e de acordo com as recomendações ergonômicas do desenho dos sistemas de turnos referidas por Knauth (1996), quanto mais tarde o turno da manhã se iniciar, melhor em termos da duração e qualidade do sono. Ora, nesse estudo, o turno da manhã se inicia às $6 \mathrm{~h}$ e termina às $14 \mathrm{~h}$, o que não é um horário "demasiado" cedo. Por outro lado, os trabalhadores residem numa área geográfica muito próxima da empresa fazendo com que o tempo gasto na deslocação casa-empresa seja relativamente 
curto. Num dos estudos realizados por Silva (2008), no qual foram entrevistados 35 trabalhadores, verificou-se que cerca de dois terços dos trabalhadores se deslocavam em seu próprio automóvel para o trabalho demorando entre 5 e 10 minutos nessa mesma deslocação.

Em relação aos trabalhadores do $3^{-0}$ turno (turno da noite fixo), estes apresentaram os scores mais baixos ao nível da "Vida Conjugal”. No entanto, o mesmo resultado não se verifica ao nível da vida familiar. Os dados também indicam a necessidade de considerar esta análise mais refinada no estudo das dimensões mais sociais do trabalho em turnos. Este resultado poderá estar relacionado com o fato de os trabalhadores do período noturno terem a possibilidade de usufruir da "parte de dia" livre (para terem um trabalho extra, estar com os amigos, prestar o apoio aos filhos, etc.) mas não estarem em casa no período no qual seria possível estar com o cônjuge (período noturno). No estudo realizado por Mott et al. (1965), os resultados apontam para que o turno noturno apenas beneficie a vida doméstica, sendo que aspectos relacionados com a proteção do cônjuge e participação nas tomadas de decisão foram avaliados negativamente.

Um aspecto presente neste estudo que parece pertinente enfatizar prende-se com a diferenciação que se optou por fazer no impacto do trabalho em turnos na vida conjugal e ao nível do desempenho dos papéis parentais (vida familiar). Embora estes construtos possam ser ambos abarcados na esfera "familiar", não parecem poder ser compreendidos como uma só dimensão, sob pena de estarem sendo omitidos aspectos importantes suscetíveis de influenciar a adaptação ao trabalho em turnos. Com estes dados, é possível também perceber que, assim como já foi referido por Handy (2010), Ljoså e Lau (2009) e Shen e Dicker (2008), o estado civil é suscetível de influenciar a adaptação a estes regimes horários.

Em termos das principais limitações do estudo, se aponta a sua natureza transversal e o fato de ter sido realizado apenas em uma organização têxtil pelo que se sugere a sua replicação a outros setores de atividade, idealmente, num design longitudinal que permita acompanhar o processo de adaptação dos trabalhadores aos diferentes turnos desde a sua entrada.

\section{Considerações finais}

Os aspectos da vida familiar e social (por exemplo, ter filhos, horário de trabalho do cônjuge, ter ou não apoio do sistema familiar) dos trabalhadores em geral e dos trabalhadores por turnos em particular estão para além da possibilidade de controle por parte das organizações, o que reforça a relevância de uma cultura organizacional participativa na gestão do tempo de trabalho. A participação dos trabalhadores ao nível da escolha dos turnos assim como no design destes, já referenciada por Baker, Roach, Ferguson e Dawson (2004) e Silva (2008), parece ser uma das formas possíveis da empresa poder proporcionar suporte nesta esfera, esperando-se assim que as dificuldades sentidas ao nível social/familiar possam ser melhor geridas.

\section{Referências}

Baer, K., Ernst, G., Nachreiner, F., \& Schay, T. (1981), Psychologische Ansaetze zur Analyse verschiedener Arbeitszeitsysteme [Psychological approaches to the analysis of various systems of working hours]. Zeitschrift für Arbeitswissenschaft, 35, 136-141.

Baer, K., Ernst, G., Nachreiner, F., \& Volger, A. (1985). Subjective bewertete Nutzbarkeit von Zeit als Hilfmittel zur Bewertung von Schichtplaenen [Subjective scaling of leisure time usefulness as means to evaluate shift schedules]. Zeitschrift für Arbeitswissenschaft, 39, 169-173.

Baker, A., Roach, G., Ferguson, S., \& Dawson, D. (2004). Shiftwork experience and the value of time. Ergonomics, 47(3), 307-317.

Bohle, P., \& Tilley, A. (1998). Early experiences of shiftwork: Influences on attitudes. Journal of Occupational and Organizational Psychology, 71, 61-79.

Bryman, A., \& Cramer, D. (2003). Análise de dados em ciências sociais: Introdução às técnicas utilizando o SPSS para Windows ( $3^{\underline{a}}$ ed. portuguesa). Oeiras: Celta

Camerino, D., Sandri, M., Sartori, S., Conway, P. M., Campanini, P., \& Costa, G. (2010). Shiftwork, work-family conflict among italian nurses, and prevention efficacy. Chronobiology International, 27(5), 1105-1123. doi: 10.3109/07420528.2010.490072.

Caplan, R. D., Cobb, S., French, J. R. P., Van Harrison, R., \& Pinneau, S. R. (1975). Job demands and worker health: Main effects and occupational differences. Washington, D.C.: National Institute for Occupational Safety and Health (NIOSH), U.S. Department of Health, Education and Welfare.

Comissão para a Igualdade no Trabalho e no Emprego. (2011). Mulheres e homens: Trabalho, emprego e vida familiar. Recuperado de http://www.cite.gov.pt/ $\mathrm{pt} / \mathrm{acite} /$ mulheresehomens $04 . \mathrm{htm}$

Costa, G. (2004). Multidimensional aspects related to shiftworkers' health and well-being. Revista de Saúde Pública, 38(supl.), 86-91. doi: http://dx.doi. org/10.1590/S0034-89102004000700013

Demerouti, E., Geurts, S., Bakker, A., \& Euwema, M. (2004). The impact of shiftwork on work - home conflict, job attitudes and health. Ergonomics, 47(9), 987-1002.

Diekmann, A., Ernst, G., \& Nachreiner, F. (1981). Auswirkungen der Schichtarbeit des Vaters auf die schulische Entwicklung der Kinder [Impact of father's shiftwork on the school development of the children], Zeitschrift für Arbeitswissenschaft, 35, 174-178.

Eisenberger, R., Huntington, R., Hutchison, S., \& Sowa, D. (1986). Perceived organizational support. Journal of Applied Psychology, 71(3), 500-507.

Field, A. (2005). Discovering statistics using SPSS (2ª ed.). Londres: Sage Publications.

Fischer, F., \& Lieber, R. (2003). Trabalho em Turnos. In R. Mendes (Org.), Patologia do Trabalho ( $2^{\underline{a}}$ ed., pp. 825-868). Rio de Janeiro: Atheneu.

Gadbois, C. (2004). Les discordances psychosociales des horaires postés: Questions en suspens. Travail Humain, 67(1), 63-85.

Handy, J. (2010). Maintaining family life under shiftwork schedules: A case study of a New Zealand petrochemical plant. New Zealand Journal of Psychology, 39(1), 29-37.

Jamal, M. (1981). Shift work related to job attitudes, social participation and withdrawal behavior: A study of nurses and industrial workers. Personnel Psychology, 34, 535-547.

Knauth, P. (1996). Designing better shift systems. Applied Ergonomics, 27(1), 39-44.

Kopelman, R., Greenhaus, J., \& Connolly, T. (1983). A model of work, family and interrole conflict: A construct validation study. Organizational Behavior and Human Performance, 32, 198-215.

Ljoså, C., \& Lau, B. (2009). Shiftwork in the Norwegian petroleum industry: Overcoming difficulties with family and social life - a cross sectional study. Journal of Occupational Medicine and Toxicology, 4(1). doi:10.1186/17456673-4-22.

Maroco, J. (2010). Análise estatística com utilização do SPSS (3- ${ }^{\underline{a}}$ ed.). Lisboa: Edições Sílabo, Lda.

Monk, T., \& Folkard, S. (1992). Making shiftwork tolerable. Londres: Taylor 
\& Francis.

Mott, P., Mann, F., McLoughlin, Q., \& Warwick, D. (1965). Shift work: The social, psychological and physical consequences. Ann Arbor, Michigan: The University of Michigan Press.

Nachreiner, F. (1998). Individual and situational determinants of shiftwork tolerance. Scandinavian Journal of Work and Environmental Health, 24(supl. 3), 35-42.

Paschoal, T., Torres, C., \& Porto J. (2010). Felicidade no trabalho: Relações com suporte organizacional e suporte social. Revista de Administração Contemporânea (Curitiba), 14(6), 1054-1072. doi: http://dx.doi.org/10.1590/ S1415-65552010000700005

Perrucci, R., MacDermid, S., King, E., Tang, C., Brimeyer, T., Ramadoss, K.,... Swanberg, J. (2007). The significance of shift work: Current status and future directions. Journal of Family and Economic Issues, 28, 600-617. doi:10.1007/s10834-007-9078-3

Pestana, M., \& Gageiro, J. (2005). Análise de dados para ciências sociais - A complementaridade do SPSS (4 $4^{\mathrm{a}}$ ed.). Lisboa: Edições Sílabo, Lda.

Presser, H. B. (1999). Toward a 24-hour economy. Science, 11(284), 1778-1779.
Saksvik-Lehouillier, Bjorvatn, B., Hetland, H., Sandal, G. M., Moen, B. E., Mageroy, N., ... Pallesen S. (2012). Individual, situational and lifestyle factors related to shift work tolerance among nurses who are new to and experienced in night work. Journal of Advanced Nursing, 1136-1146. doi: 10.1111/j.1365-2648.2012.06105.x.

Shen, J., \& Dicker, B. (2008). The impact of shiftwork on employees. The International Journal of Human Resource Management, 19(2), 392-405.

Silva, C. F., Azevedo, M. H., \& Dias, M. R. (1994). Estudo Padronizado do Trabalho por Turnos (Versão Experimental). Braga: Departamento de Psicologia da Universidade do Minho e Coimbra: Faculdade de Medicina da Universidade de Coimbra.

Silva, I. S. (2008). Adaptação ao trabalho por turnos (Dissertação de Doutorado). Recuperado de http://hdl.handle.net/1822/7723

Smith, L., \& Folkard, S. (1993). Is a 'safer' permanent night shift practicable? Ergonomics, 36, 317-3

West, S., Mapedzahama, V., Ahern, M., \& Rudge, T. (2012). Rethinking shiftwork: Mid-life nurses making it work. Nursing Inquiry, 19(2), 177-187. doi: 10.1111/j.1440-1800.2011.00552.x.

Ana Isabel Ferreira, mestre em Psicologia do Trabalho, das Organizações e dos Recursos Humanos pela Universidade do Minho (UM), Portugal, é técnica de Recursos Humanos numa empresa de retalho em Portugal. E-mail: anaiferreira@gmail.com

Isabel Soares Silva, doutora em Psicologia do Trabalho e das Organizações pela Universidade do Minho (UM), Portugal, é professora auxiliar na Escola de Psicologia da Universidade do Minho (EPsi/UM), Portugal. Endereço para correspondência: Universidade do Minho, Escola de Psicologia, Departamento de Psicologia Aplicada, Campus de Gualtar, 4710-057 Braga, Portugal. Telefone: (+351) 253604 220. Fax: (+351) 253 604221 E-mail: isilva@psi.uminho.pt 